\title{
Reintegro al trabajo en la post-pandemia COVID-19: un desafío emergente para la salud ocupacional
}

\section{Return to work in the post COVID-19 pandemic: an emerging challenge for occupational health}

\section{Estimado editor:}

https://doi.org/10.52808/bmsa.7e5.612.028

La pandemia por SARS-CoV-2 crea nuevos desafíos para la salud ocupacional, al desviar la atención del reintegro a la actividad laboral, después de cursar la enfermedad, durante un brote, lidiar con el encierro y tener especialmente en cuenta a los trabajadores con vulnerabilidades (Shaw et al., 2020; Taylor et al., 2020).

Recomendamos considerar tres aspectos diferentes de la salud ocupacional durante una pandemia. En primer lugar, para la mayoría de los trabajadores con alto riesgo de COVID-19 grave, los problemas de discapacidad laboral y reintegro del trabajo nunca habían ocurrido antes de la epidemia. Recomendaciones como el distanciamiento social, distanciamiento físico y el uso de una mascarilla son muy recomendables para protegerse contra las infecciones, pero pueden no ser suficientes para que algunas personas puedan reanudar el trabajo. Por lo tanto, la toma de decisiones requiere evaluaciones integrales individuales de la afección médica subyacente, el riesgo de contaminación por SARS-CoV-2 asociado con el trabajo regular o el teletrabajo y las oportunidades de vacunación.

La segunda situación se refiere a los trabajadores que han sufrido COVID-19. Los estudios preliminares sugieren que la duración prolongada de la recuperación está relacionada con la gravedad alta (Taylor et al., 2020), pero esto sigue siendo un tema de debate para los pacientes que padecen COVID-19 prolongado (Huang et al., 2021; Carfì et al., 2020), una condición para la cual los efectos siguen siendo desconocidos. Cualquier recuperación prolongada debe considerarse un signo potencial de COVID-19 prolongado. Estos síndromes de larga duración ocurren en pacientes con síntomas graves, pero también se han informado independientemente de la gravedad de la fase aguda, la hospitalización y la recepción de oxígeno-terapia (Carfì et al., 2020). En la actualidad, investigadores de todo el mundo están investigando estos síndromes. Será necesario implementar estrategias que promuevan el regreso al trabajo de estos trabajadores y podrían ser similares a los programas desarrollados para otras afecciones crónicas.

Finalmente, las consecuencias de la epidemia deben evaluarse en el tiempo para las personas que sufrieron limitaciones funcionales antes del COVID-19 ya que su condición física y mental puede verse modificada por la epidemia y, específicamente, las consecuencias del encierro (Chudasama et al., 2020).

En todas estas situaciones, los contextos médico, social, financiero y laboral son elementos clave. Además de una evaluación médica, el uso de escalas como Work Ability Index (Tuomi, 1997) o Work Productivity and Activity Impedirment (Reilly et al., 1993) puede ayudar a realizar un seguimiento a largo plazo y proporcionar información sobre el trabajo, capacidad y carga de trabajo. También ofrece una perspectiva de volver a lo básico, instando a los políticos a avanzar hacia una política de trabajo decente para todos, como defiende el Objetivo de Desarrollo Sostenible (ODS) 8 de las Naciones Unidas.

Sin más, se despide

Roberto Carlos Dávila Morán 1

${ }^{1}$ Universidad Privada del Norte (UPN). Lima, Perú Autor de correspondencia: rdavila430@gmail.com https://orcid.org/0000-0003-3181-8801

\section{Referencias}

Carfì A., Bernabei R., \& Landi, F. (2020). Gemelli against COVID-19 post-acute care study group. Persistent symptoms in patients after acute COVID-19. JAMA. 324(6); 603-605.

Chudasama Y.V., Gillies C.L., Zaccardi F., Coles B., Davies M.J., Seidu S, \& Khunti K. (2020). Impact of COVID-19 on routine care for chronic diseases: a global survey of views from healthcare professionals. Diabetes \& Metabolic Syndrome: Clinical Research \& Reviews. 14(5):965-967.

Huang C., Huang L., Wang,Y., Li X., Ren L., GuX., \& Cao B. (2021). 6-month consequences of COVID-19 in patients discharged from hospital: a cohort study. The Lancet. 397(10270):220-232.

Shaw W.S., Main C.J., Findley P.A., Collie A., Kristman V.L. \& Gross D.P. (2020). Opening the workplace after COVID-19: what lessons can be learned from return-to-work research? Journal of Occupational Rehabilitation. 30:299-302.

Taylor T., Das R., Muelle K., Pransky G., Christian J., Orford R. \& Blink R. (2020). Safely returning America to work: part I: general guidance for employers. Journal of occupational and environmental medicine. 62(9):771-779. 